(C) 2013 Elsevier B.V. This manuscript version is made available under the CC-BY-NC-ND 4.0 license (http:// creativecommons.org/licenses/by-nc-nd/4.0/)

\title{
A new potential nano-oncological therapy based on polyaminoacid nanocapsules
}

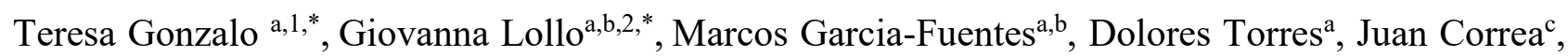
Ricardo Riguerac ${ }^{\mathrm{c}}$, Eduardo Fernandez-Megia ${ }^{\mathrm{c}}$, Pilar Calvo ${ }^{\mathrm{d}}$, Pablo Avilés ${ }^{\mathrm{d}}$, Maria José Guillén ${ }^{\mathrm{d}}$, Maria José Alonso ${ }^{\mathrm{a}, \mathrm{b} \dagger}$

* Authors who equally contributed to this work; ${ }^{\dagger}$ Author for correspondence

${ }^{a}$ Department of Pharmaceutics and Pharmaceutical Technology, School of Pharmacy, Campus Vida, University of Santiago de Compostela, 15782 Santiago de Compostela, Spain; ${ }^{b}$ Center for Research in Molecular Medicine and Chronic Diseases (CIMUS) Campus Vida University of Santiago de Compostela, 15782 Santiago de Compostela, Spain; 'Department of Organic Chemistry and Center for Research in Biological Chemistry and Molecular Materials, Campus Vida, University of Santiago de Compostela, 15782 Santiago de Compostela, Spain; ${ }^{\text {PharmaMar }}$ S.A., Avda de los Reyes, 1 Pol. Ind. La Mina 28770 Colmenar Viejo (Madrid) Spain.

éPresent address: Ambiox Biotech, Avda Buendia 11, 19005 Guadalajara (Madrid), Spain.

i Present address: Université d'Angers, Angers F-49100, INSERM U1066 MINT, MIcro et Nanomédecines biomiméTiques, IBS-CHU ANGERS, 4 rue Larrey, 49933 Angers Cedex 9, France.

${ }^{\dagger}$ Corresponding author: Maria J. Alonso

E-mail address: mariaj.alonso@usc.es 
Abstract

A critical objective in cancer therapy is to reduce the systemic toxicity through the modification of the biodistribution of anticancer drugs. Herein, we disclose a new biodegradable nanocarrier, polyglutamic acid (PGA) nanocapsules, and present the in vivo pharmacokinetics/toxicity proof-ofconcept for the anticancer drug plitidepsin. These novel nanocapsules were prepared using a modified solvent displacement technique where the polyaminoacid was electrostatically deposited onto the lipid core. The nanocapsules exhibited an average size of $200 \mathrm{~nm}$, a negative zeta potential and a great capacity for the encapsulation of plitidepsin (encapsulation efficiency above 90\%). In addition, the nanocapsules could be freeze-dried and showed an adequate stability profile upon storage. Finally, the in vivo proof-of-concept studies performed in mice indicated that the encapsulation provided the drug with a prolonged blood circulation and a significantly reduced toxicity. In fact the maximum tolerated dose of the nanoencapsulated drug was more than 3 times that of the reference formulation (Cremophor ${ }^{\circledR}$ EL plitidepsin solution). Overall, beyond the value of this specific formulation, the work reported here represents the evidence of the potential of polyaminoacid nanocapsules in nano-oncological therapy.

Keywords: nanomedicines, long-circulating nanocarriers, nanocapsules, polyglutamic acid, cancer. 
The clinical use of most anticancer drugs is associated to severe side effects and poor quality of life for the patients. These effects are mainly related to the indiscriminate systemic biodistribution of anticancer drugs and their accumulation in non-target tissues [1]. In addition, the excipients used for formulating hydrophobic anticancer drugs may contribute to their systemic toxicity [2]. The limitations of these conventional formulations have stimulated an intensive search for nanocarriers capable of reducing the toxicity of anticancer drugs $[3,4]$. The advances achieved so far are illustrated by the commercialization and clinical development of a significant number of formulations based on liposome [5], nanoparticle [6, 7], conjugates [8] and micelle technologies [9]. However, the need to further improve the efficacy/toxicity profile of anticancer drugs, and thus the necessity to develop alternative delivery vehicles, persists. Within this frame the design of polymer nanocapsules might, in our understanding, offer specific advantages [10]. First, the oily core of polymeric nanocapsules is an ideal environment for the encapsulation of hydrophobic antitumor drugs at high payload [11, 12]. Second, the polymeric shell can be conveniently designed in order to improve the biodistribution profile of the nanocapsules, either extending the half-life of the encapsulated drug or even targeting the drug to specific cells [13]. Third, nanocapsules can be formulated to be stable over the time and also to be freeze-dried in order to further prolong their stability profile [11]. Altogether these positive features render nanocapsules as an attractive formulation strategy for improving the efficacy/toxicity balance of anti-cancer drug candidates.

Among the different polymers described until now for the design of anticancer drug nanocarriers, PEG and its derivatives have been the most widely investigated [14]. The PEG hydrophilic character makes it a suitable material for preventing the uptake of nanocarriers by the mononuclear phagocytic system (MPS) and enhancing the circulation time of associated drugs [15]. However, current efforts are being conducted to the search for new polymers, which might provide the nanocarriers with specific advantages beyond the long circulating properties. In this regard, polyaminoacids, and in particular polyglutamic acid (PGA), are at the front line of attention because of their attractive safety profile. PGA, an anionic polyaminoacid composed of naturally occurring L-glutamic acid linked by peptide bonds, is known to be biocompatible and biodegradable [16, 17]. Moreover, its long-circulating behaviour and enhanced accumulation at the tumour site, has been made evident for PGA-paclitaxe ${ }^{\circledR}$ conjugates, currently in phase III clinical trials [18, 19]. Alternatively, cisplatin loaded micelles consisting of PGA and methoxypoly(ethylene glycol) are being investigated for NSCL lung cancer showing promising antitumor results [20].

Taking this information into account, the main goal of this work has been to design and develop a new nanocapsule-type delivery platform based on the use of PGA. Besides the long circulating properties, the specific advantages expected for PGA nanocapsules rely on their theoretical ability to encapsulate significant amounts of hydrophobic drugs and also on their enhanced in vitro and in vivo stability. We have also compared the behaviour of PGA nanocapsules with that of polyethylenglycol-grafted PGA (PGA-PEG) nanocapsules in order to establish the effective value of PGA as a material for the development of long-circulating nanocapsules [21, 22]. The potential of this new nanotechnology platform has been assessed using the anticancer drug plitidepsin. Plitidepsin is a highly hydrophobic cytostatic active ingredient originally isolated from the marine tunicate Aplidium Albicans, and now manufactured synthetically by PharmaMar S.A. as a potential treatment for a variety of cancers [23]. Following extensive in vitro characterization of the unloaded and plitidepsin-loaded PGA and PGA-PEG nanocapsules, we assessed the in vivo proof-of-principle for their capacity to improve the maximum tolerated dose and the pharmacokinetic parameters of the drug. 
Plitidepsin was kindly provided by PharmaMar S.A. (Spain). Poloxamer (Pluronic F-68®), benzalkonium chloride (BKC) and poly-L-glutamic acid (PGA) (Mw 15-50 KDa) were purchased from Sigma-Aldrich (Spain). Miglyol ${ }^{\circledR} 812$, which is a neutral oil formed by esters of caprylic and capric fatty acids and glicerol, was donated by Sasol Germany GmbH (Germany). Poly(ethyethylene glycol)-stearate of a degree of polymerization of 40 hence forth designated as PEG 40-stearate [Simulsol M52] (Seppic, France). The surfactant Epikuron 170, which is a phosphatidylcholine-enriched fraction of soybean Lecithin, was donated by Cargill (Spain).

\section{Synthesis of PGA-PEG}

PGA (100 mg, $0.662 \mathrm{mmol}$ of repetition unit, $M_{\mathrm{n}} 10,900$ by multi angle laser light scattering, degree of polymerization 72) and MeO-PEG-NH $2\left(43.6 \mathrm{mg}, 8.4 \mu \mathrm{mol}, M_{\mathrm{n}} 5,219, M_{\mathrm{w}} 5,242\right.$ by MALDITOF) were dissolved in $\mathrm{H}_{2} \mathrm{O}(2 \mathrm{~mL})$. 1-Hydroxy-benzotriazole (11 mg, $\left.84 \mu \mathrm{mol}\right)$ and 1-ethyl-3-(3dimethylaminopropyl) carbodiimide hydrochloride $(13 \mathrm{mg} 84 \mu \mathrm{mol})$ were added and the reaction was allowed to stir overnight. The resulting product was purified by ultrafiltration (Amicon YM30, $15 \times 50 \mathrm{~mL} \mathrm{H}_{2} \mathrm{O}$ ) to obtain final PGA-PEG (degree of PEGylation $1.17 \%$ by ${ }^{1} \mathrm{H}$ NMR, $87 \%$ yield, $24 \% \mathrm{w} / \mathrm{w}$ of PEG). ${ }^{1} \mathrm{H}$ NMR $\left(500 \mathrm{MHz}, \mathrm{D}_{2} \mathrm{O}\right): \delta$ 4.51-4.16 (m, $\left.72 \mathrm{H}\right), 3.89-3.57(\mathrm{~m}, 523 \mathrm{H}), 3.43$ (s, 3.5H), 2.65-1.84 (m, 288H).

\section{Preparation of PGA and PGA-PEG nanocapsules}

PGA nanocapsules were prepared by the solvent displacement technique [24]. Briefly, the organic phase composed of plitidepsin $(1.2 \mathrm{mg}), 0.125 \mathrm{~mL} \mathrm{Miglyol}{ }^{\circledR} 812,7 \mathrm{mg}$ of the cationic surfactant BKC, $30 \mathrm{mg}$ Epikuron 170 in $0.5 \mathrm{~mL}$ of ethanol and $9 \mathrm{~mL}$ acetone was added onto an aqueous phase composed of the non-ionic surfactant Pluronic $188(0.25 \% \mathrm{w} / \mathrm{v})$ and the polymer PGA or PGA-PEG $(10 \mathrm{mg})$. Nanocapsules were formed immediately upon the mixture of both phases. The organic solvents were evaporated under vacuum. Unloaded nanocapsules were prepared by the same method, in absence of plitidepsin in the organic phase.

Nanoemulsions and PEG-coated nanoemulsions were also prepared and used as controls in order to prove the value of the polymeric coating. They were obtained by the same technique as described above, but without adding PGA or PGA-PEG to the external water phase. Instead, PEG-surface modified nanoemusions were formed by including $6.42 \mathrm{mg}$ of PEG-stearate to the organic phase, the calculated amount of modified lipid required for having the same amount of PEG than with the PGA-PEG coating.

\section{Physicochemical Characterization of PGA and PEG-PGA nanocapsules}

PGA and PGA-PEG nanocapsules were characterized with regard to size, zeta potential and morphology. Particle size and polydispersity index were determined by photon correlation spectroscopy (PCS) after dilution with bi-distilled water. Analyses were carried out at $25^{\circ} \mathrm{C}$ with an angle detection of $173^{\circ}$. The zeta potential values were calculated from the mean electrophoretic mobility values, as determined by laser Doppler anemometry (LDA). For LDA measurements, samples were diluted with $\mathrm{KCl} 1 \mathrm{mM}$ and placed in an electrophoretic cell. PCS and LDA analysis were performed in triplicate using a NanoZS ${ }^{\circledR}$ (Malvern Instruments, Malvern, UK).

The morphology of nanocapsules was studied by Transmission Electron Microscopy (TEM) using a Philips CM-12 (FEI Company, Eindhoven, The Netherlands), following negative staining with a phosphotungstic acid solution $(2 \%, w / v)$ and immobilization on copper grids with Formvar ${ }^{\mathbb{R}}$.

\section{Plitidepsin encapsulation and release studies}

The encapsulation efficiency of plitidepsin in the nanocapsules was determined by the difference between the amount of plitidepsin in the supernatant and the total amount in the nanocapsules. Plitidepsin content in the supernatant was established upon isolation of the drug from the nanocapsules by ultrafiltration in Amicon columns (Amicon Ultra-4, 100000MWCO, Millipore, 
Spain). Then, samples of the supernatants or the nanocapsule suspension (for the total plitidepsin content) were dissolved in acetonitrile and analysed by HPLC.

The in vitro drug release from the nanocapsules was performed in PBS $(0.01 \mathrm{M})$ with $4 \%$ bovine serum albumin (BSA) under sink conditions. The concentration of plitidepsin in the medium was 1 $\mu \mathrm{g} / \mathrm{mL}$ which corresponds to sink conditions [25]. Samples were incubated at $37^{\circ} \mathrm{C}$ and withdrawn at appropriate time intervals $(15 \mathrm{~min}, 1 \mathrm{~h}, 3 \mathrm{~h}, 6 \mathrm{~h}$ and $24 \mathrm{~h})$. Total plitidepsin content was determined by HPLC after dissolving a portion of each sample in acetonitrile, followed by mild centrifugation ( $3 \mathrm{~min}, 4000 \mathrm{~g}$ ) to precipitate suspended proteins. Released plitidepsin was calculated upon isolation of the free drug by ultracentrifugation $\left(27400 \mathrm{~g}, 1 \mathrm{~h}, 15^{\circ} \mathrm{C}\right)$. The supernatant was then analysed by HPLC following the same treatment described above for total plitidepsin content. The HPLC system consisted of an Agilent 1100 series instrument equipped with UV detector set at $225 \mathrm{~nm}$. The analytic method for plitidepsin quantification has been previously reported by PharmaMar S.A. [26].

\section{Stability of plitidepsin-loaded PGA nanocapsules during storage}

The stability of plitidepsin-loaded PGA nanocapsules was evaluated under storage conditions for 8 weeks at $4^{\circ} \mathrm{C}$, room temperature and $37^{\circ} \mathrm{C}$. Three parameters were assessed at different time points: (i) macroscopic aspect (presence of aggregated, cream formation, changes in color, etc.); (ii) particle size, polydispersity and zeta potential; (iii) plitidepsin concentration in the preparation and encapsulation efficiency. All these characteristics were determined as described above.

\section{Freeze-drying studies of plitidepsin-loaded PGA nanocapsules}

Blank and plitidepsin-loaded PGA nanocapsules at different concentrations between 1 and $0.5 \%$ $\mathrm{W} / \mathrm{v}$ were freeze-dried by immersion in liquid nitrogen in the presence of trehalose $(10 \% \mathrm{w} / \mathrm{v})$. The freeze-drying programme consisted in an initial drying step at $-35^{\circ} \mathrm{C}$, and secondary drying where temperature was finally equilibrated at $20^{\circ} \mathrm{C}$ over a period of $60 \mathrm{~h}$ (Labconco Corp., USA). PGA nanocapsules were resuspended by adding $1 \mathrm{~mL}$ of ultrapure water to the freeze-dried cake followed by gentle agitation. The size and polydispersity of the resuspended nanocapsules was evaluated by PCS.

\section{In vivo studies \\ Animals}

Studies were performed with CD-1 male mice (Harlan Interfauna Iberica S.L., Barcelona, Spain), housed Makrolon cages (10 animals/cage). Animals were subjected to preliminary observation and to an acclimatisation period. The animal house was maintained at $21-23^{\circ} \mathrm{C}$, with $35-55 \%$ relative humidity. Illumination was controlled to allow for 12 hours of light and 12 hours of darkness. All animals were observed for morbidity/mortality throughout the whole assay.

\section{Pharmacokinetic evaluation}

Pharmacokinetic studies of plitidepsin were performed upon i.v. administration of different formulations to CD-1 mice $(n=36)$. The formulations tested were: PGA nanocapsules, PGA-PEG nanocapsules, nanoemulsions and PEG-coated nanoemulsions. Mice of 20-25 g weight were selected for these studies. A volume of $250 \mu \mathrm{l}$ of the different plitidepsin formulations were injected in the lateral vein of the tail. The injected plitidepsin dose was $0.1 \mathrm{mg} / \mathrm{kg}$ for nanoemulsion and PEGylated nanoemulsion and $0.4 \mathrm{mg} / \mathrm{kg}$ for PGA and PGA-PEG nanocapsules. Blood samples were collected in EDTA microtubes at the following times postinfusion: $5 \mathrm{~min}, 15 \mathrm{~min}, 30 \mathrm{~min}, 1 \mathrm{~h}$, $3 \mathrm{~h}, 6 \mathrm{~h}, 24 \mathrm{~h}$ and $48 \mathrm{~h}$. The samples were centrifuged at $4000 \mathrm{~g}$ for 15 minutes at approximately $5^{\circ} \mathrm{C}$. The resulting plasma was frozen at $-20^{\circ} \mathrm{C}$ until analysis by HPLC-MS/MS.

Plitidepsin concentrations were quantified by HPLC-MS/MS after solid-liquid extraction with a mixture of tert-butyl methyl ether (TBME): hexane $(1: 1, \mathrm{v} / \mathrm{v})$. The pharmacokinetic parameters of plitidepsin were performed using a non-compartmental pharmacokinetic method with a 
WinNonlin ${ }^{\mathrm{TM}}$ Professional Version 4.01 (Pharsight Corporation, Mountain View, CA, USA). The AUC values given are normalized to the dose given.

\section{Toxicological evaluation}

The Maximun Tolerated Dose (MTD) of different formulations was evaluated after i.v. administration. The formulations tested were: plitidepsin-loaded PGA nanocapsules, PGA-PEG nanocapsules, nanoemulsions, PEG-coated nanoemulsions and the reference formulation (Cremophor ${ }^{\mathbb{B}}$ EL/Ethanol/Water 15/15/70 w/w/w solution). The formulations at different plitidepsin doses $(0.2-1 \mathrm{mg} / \mathrm{kg})$ were administered as a single i.v. bolus in the lateral vein of the tail. Groups of 8 animals were used for each dose level. A control group consisting of 8 animals was administered with non-loaded nanocapsules, to evaluate potential toxicity. The animals were weighted at the start of the study, twice a week, and before being sacrificed. Mortality checks were performed at least once a day during the whole assay (14 days). Any mouse showing signs of extreme weakness, toxicity or in a moribund state was sacrificed. The animals were monitored at least once a day during the whole assay and any clinical responses were carefully noted. The observations included changes in weight, skin and fur, eyes and mucous membranes, respiratory, circulatory, central nervous and autonomic nervous systems, somatomotor activity and behavior.

\section{Results and Discussion}

This article describes for the first time the design and development of a novel drug nanocarrier based on PGA nanocapsules. The rationale for the selection of PGA for the formation of the nanocapsule's shell was its biocompatibility and its potential ability to provide stealth properties to the carrier [17, 27, 28]. As an alternative, a PEG-grafted PGA (PGA-PEG) copolymer was also investigated. Herein, we discuss the development and physicochemical characterization of these nanocarriers, their capacity for the encapsulation and delivery of the anticancer drug plitidepsin and, finally, their ability to modify the toxicity and pharmacokinetics profiles of this drug.

\section{Preparation and characterization of unloaded and plitidepsin-loaded PGA and PGA-PEG nanocapsules}

Nanocapsules were obtained according to a modified solvent displacement technique where the coating polymer is deposited onto the oily core by electrostatic interaction. A similar approach has been reported by our group for the formation of positively charged chitosan nanocapsules [24, 29]. However, in this case, it was necessary to define a technical approach that would facilitate the interaction between the negatively charged oily core and the acidic polymer coating. This approach was based on the use of cationic surfactants, which may potentially act as bridges between the oily core and the polymer coating (Figure 1).
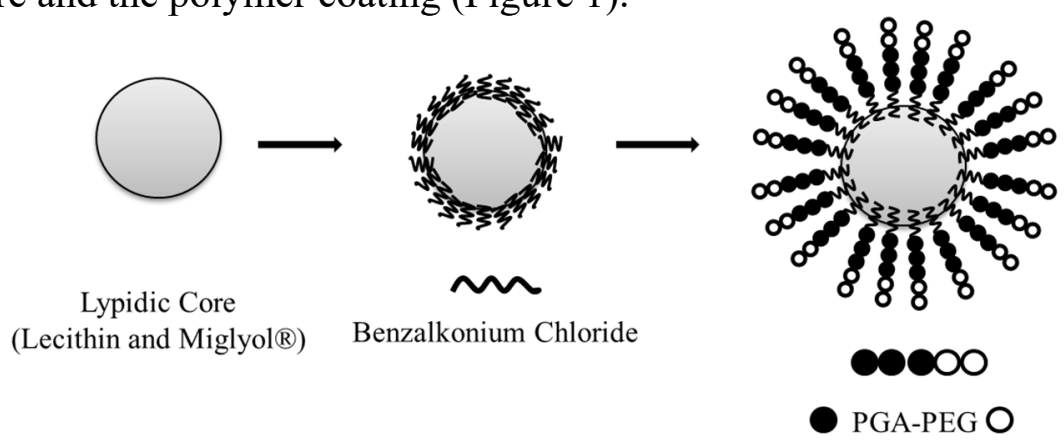

\section{Figure 1: Illustration of the structure of PGA and PGA-PEG nanocapsules.}

In a first instance, we explored a variety of poloxamines (Tetronic 908, Tetronic 904, Tetronic and 901, BASCOM, Brussels, Belgium, HLB $=2.5,14.5$ and 30.5, respectively) because of the presence of amine groups in their polymer backbone, which could potentially become protonated. However, irrespective of the amount of poloxamine, we found that the introduction of increasing 
amounts of poloxamine in the system did not lead to a positive zeta potential nanoemulsions. In a second series of studies, we explored the utility of stearylamine, a cationic phospholipid extensively used in the formulations of liposomes and emulsions [30]. Unfortunately, under a number of different processing conditions (involving varying volumes of organic/aqueous phase, heating organic phase at $70^{\circ} \mathrm{C}$, or using ethanol, ethyl acetate or dichloromethane as organic solvents) this surfactant led to the formation of aggregated particles rather than nanocapsules. Lastly, we studied the behavior of surfactants such as benzalkonium chloride and cetylpyridinium chloride, which had already been used in the formation of nanoparticles [31]. The introduction of these surfactants led to the formation of positively charged surfaces, which facilitated the further adhesion of the PGA shell while maintaining the small size and stability of the oily nanodroplets.

The mean particle size of unloaded PGA and PGA-PEG nanocapsules prepared using benzalkonium chloride was approximately $200 \mathrm{~nm}$, corresponding to a monomodal and narrow size distribution (polydispersity index $=0.1$ ). Both unloaded PGA and PGA-PEG nanocapsules exhibited a negative charge $(-40 \mathrm{mV}$ and $-28 \mathrm{mV}$ respectively), whereas the control nanoemulsion had a high positive charge $+38 \mathrm{mV}$ ). This charge inversion is an indication of the electrostatically-driven formation of the PGA shell. The reduced negative charge observed for the PEGylated nanocapsules could be associated to the known PEG shielding effect [32]. In addition to the nanocapsules and control nanoemulsion, a PEGylated nanoemulsion of the same composition as the control but containing PEG-stearate was also formulated for further comparative analysis. As expected, the introduction of PEG in the same amount as in the PGA backbone led to a reduction of the positive charge $(+26$ $\mathrm{mV}$ ), although in this case no charge inversion was observed. Plitidepsin-loaded nanocapsules showed similar physicochemical properties compared to the unloaded ones (Table 1).

Table 1: Characterization of size and zeta potential of unloaded and plitidepsin-loaded nanocapsules and nanoemulsions (Mean \pm S.D.; n=3). E.E.: Encapsulation efficiency; P.I: Polydispersity Index, NCs: nanocapsules; NE: nanoemulsion.

\begin{tabular}{lccccc}
\hline Prototype & $\begin{array}{c}\text { Plitidepsin } \\
\text { conc. }(\mathbf{m g} / \mathbf{m L})\end{array}$ & $\begin{array}{c}\text { Size } \\
\mathbf{( n m )}\end{array}$ & P.I. & $\begin{array}{c}\text { Zeta potential } \\
\mathbf{( m V )}\end{array}$ & E.E. (\%) \\
\hline NE & - & $207 \pm 7$ & 0.1 & $+38 \pm 1$ & - \\
\multirow{2}{*}{ PEG NE } & 0.12 & $203 \pm 7$ & 0.1 & $+40 \pm 1$ & 95 \\
& - & $200 \pm 3$ & 0.1 & $+26 \pm 1$ & - \\
PGA NCs & 0.12 & $203 \pm 5$ & 0.1 & $+28 \pm 3$ & 98 \\
& - & $202 \pm 5$ & 0.1 & $-40 \pm 5$ & - \\
PEG-PGA NCs & 0.12 & $183 \pm 6$ & 0.1 & $-38 \pm 1$ & 99 \\
& - & $191 \pm 4$ & 0.1 & $-28 \pm 4$ & - \\
& 0.12 & $201 \pm 5$ & 0.1 & $-28 \pm 3$ & 98 \\
\hline
\end{tabular}

On the other hand, TEM images confirmed the values of particle size for PGA and PGA-PEG nanocapsules measured by PCS, and the homogeneity of the particle size distribution (Figure 2). Moreover, TEM images provided evidence of the rounded and regular morphology as well as the core-shell type of structure. 

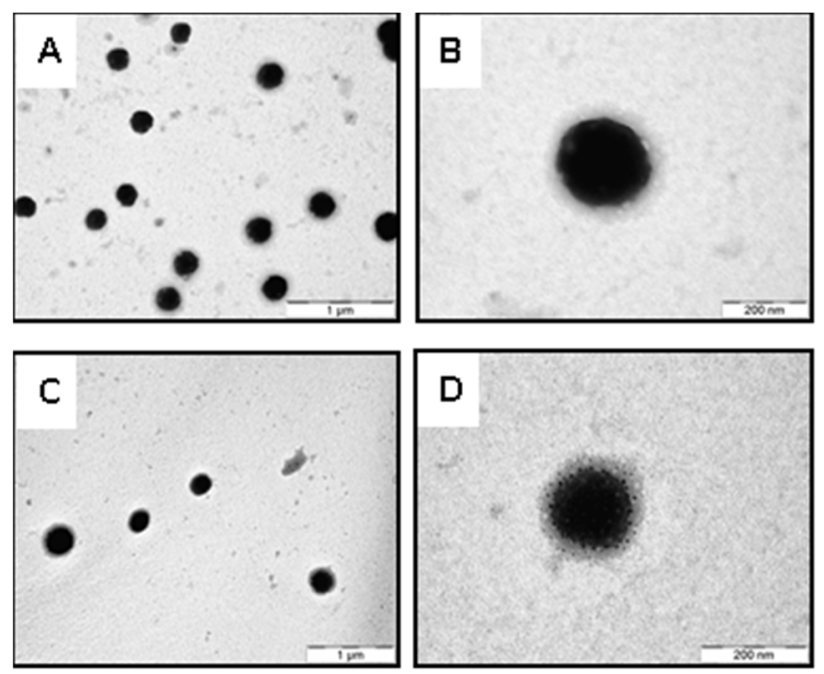

Figure 2: TEM images of PGA and PGA-PEG nanocapsules containing plitidepsin. PGA nanocapsules (A, B); PGA-PEG nanocapsules: (C, D).

\section{Plitidepsin encapsulation and release from PGA and PGA-PEG nanocapsules}

The encapsulation efficiency of plitidepsin into PGA and PEG-PGA nanocapsules was very high (98-99\%, final loading $0.54 \%$ weight of plitidepsin/weight of total components) (Table 1). This result was attributed to the great affinity of the drug for the selected oily core. The release rate of the drug was also monitored upon encapsulation of PGA and PGA-PEG nanocapsules in simulated biological media (PBS with BSA $4 \% \mathrm{w} / \mathrm{w}$ ) at $37^{\circ} \mathrm{C}$, under sink conditions. Despite de fact that nanocapsules were stable in this medium (data not shown), the results in Figure 3, indicate that PGA and PEG-PGA nanocapsules released $60 \%$ of their cargo during the first hour and no further release was observed for the remaining time of the experiment $(24 \mathrm{~h})$. This biphasic release profile, has been previously observed for other types of nanocapsules [11,33]. The initial burst has been typically associated to the partition of the drug between the oily cores and the great volume of the external aqueous phase. Even though these results cannot be extrapolated to the in vivo situation, the fact that a significant fraction of the drug remained encapsulated despite the "sink conditions", is an indication of the high affinity of plitidepsin for the oily core and/or the shell of the nanocapsules. Finally, the presence of PEG in the nanocapsules shell did not affect the release properties of the nanocarriers (Figure 3 ).

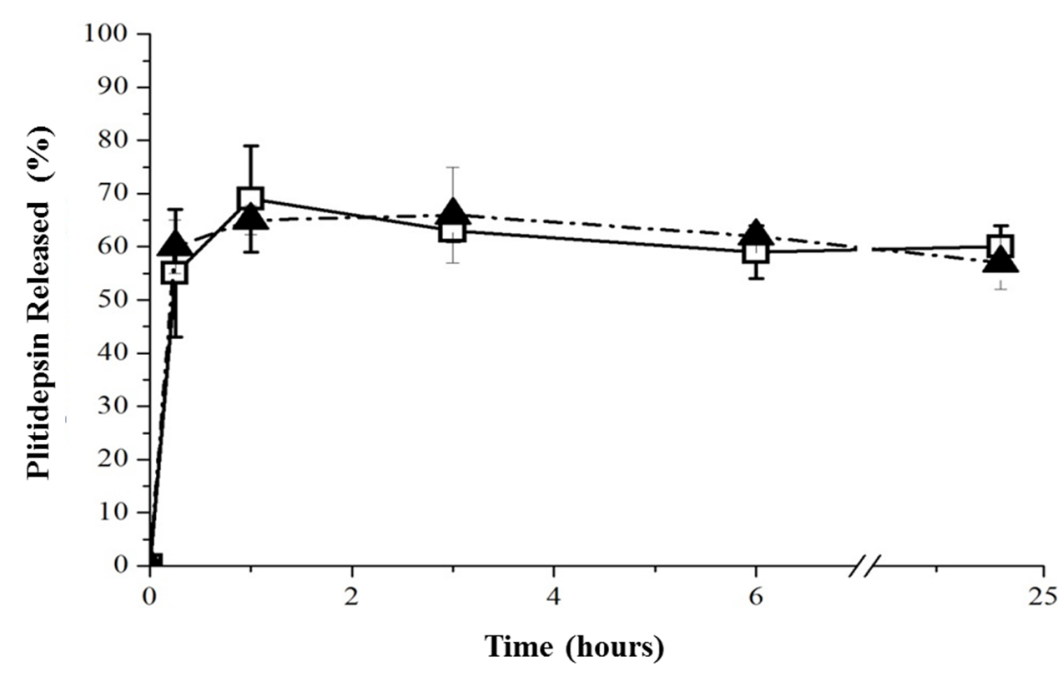

Figure 3: In vitro drug release from plitidepsin-loaded nanocapsules in PBS with BSA (4\% w/w) medium. PGA nanocapsules $(\square)$; PGA-PEG nanocapsules $(\boldsymbol{\Delta})$. (Mean \pm S.D.; $n=3$ ). 
Stability of plitidepsin-loaded PGA nanocapsules upon storage.

The stability of plitidepsin-loaded PGA nanocapsules was assessed upon storage at different temperatures $\left(4^{\circ} \mathrm{C}\right.$, room temperature and $\left.37^{\circ} \mathrm{C}\right)$ for up to 8 weeks. The parameters determined at different time points were: physicochemical properties, i.e., particle size, polydispersity and zeta potential, and plitidepsin stability and encapsulation efficiency. The preservation of the nanocapsules particle size and polydispersity is critical to ensure that the nanocarrier maintains the biodistribution properties [34]. Finally, the stability of plitidepsin and its encapsulation efficiency are important to ensure reproducible pharmacokinetics and pharmacological potency over time.

The particle size evolution of PGA nanocapsules over the time is shown in Figure 4 . At $4^{\circ} \mathrm{C}$ and room temperature, no significant differences on the mean particle size and polydispersity index of plitidepsin-loaded PGA were observed during the 8-week study. Zeta potential determinations performed in the different samples confirmed that the surface electrical charge of the nanocarriers did not change over time, another indication of chemical and physicochemical stability of the system (data not shown).

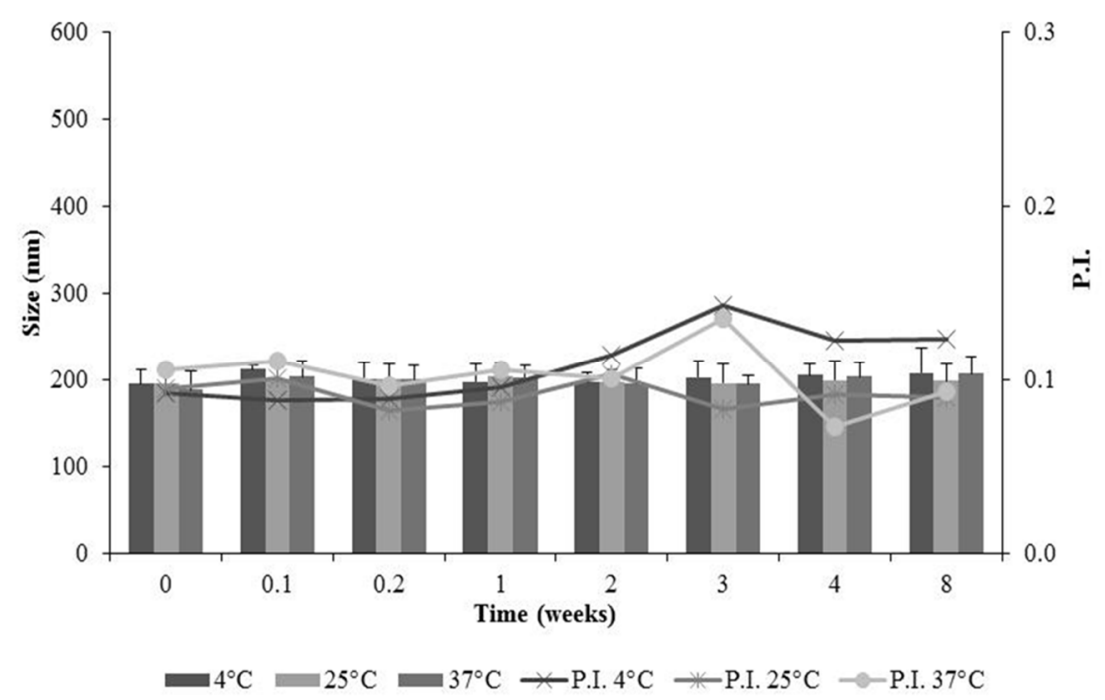

Figure 4: Stability upon storage of plitidepsin-loaded PGA nanocapsules at different temperatures. (Mean \pm S.D.; n=3) P.I: Polydispersity Index.

Finally, with regard to the stability of the encapsulated plitidepsin, the results showed that after an 8 week-storage period at $4^{\circ} \mathrm{C}$, the drug content and the encapsulation efficiency of the formulation were maintained. Overall these results indicate that PGA nanocapsules have excellent stability characteristics.

\section{Freeze-drying studies of plitidepsin-loaded PGA nanocapsules}

For better handling and for storing for prolonged periods of time, a freeze-dried formulation of PGA nanocapsules was developed. Non-loaded and plitidepsin-loaded PGA nanocapsules suspensions at different concentrations $(1,0.75,0.5 \% \mathrm{w} / \mathrm{v}$ final concentration) were mixed with trehalose $(10 \%$ $\mathrm{w} / \mathrm{v}$ final concentration) and freeze-dried. The results indicated that PGA nanocapsules could be freeze-dried at any of the concentrations tested with minimum changes in their particle size. The same behaviour was observed for the formulations containing plitidepsin (data not shown).

\section{Pharmacokinetic evaluation}

As indicated, in this work plitidepsin was encapsulated into PGA and PGA-PEG nanocapsules with the final objective of enhancing the plasma residence time of the drug, a necessary step in promoting its passive targeting to solid tumors [35]. To assess this, the pharmacokinetic profile of plitidepsin administered in the PGA and PGA-PEG nanocapsules formulations in comparison to 
that of the control emulsions was studied in healthy mice. The plasmatic drug concentrations following i.v. administration of the different formulations are shown in Figure 5.

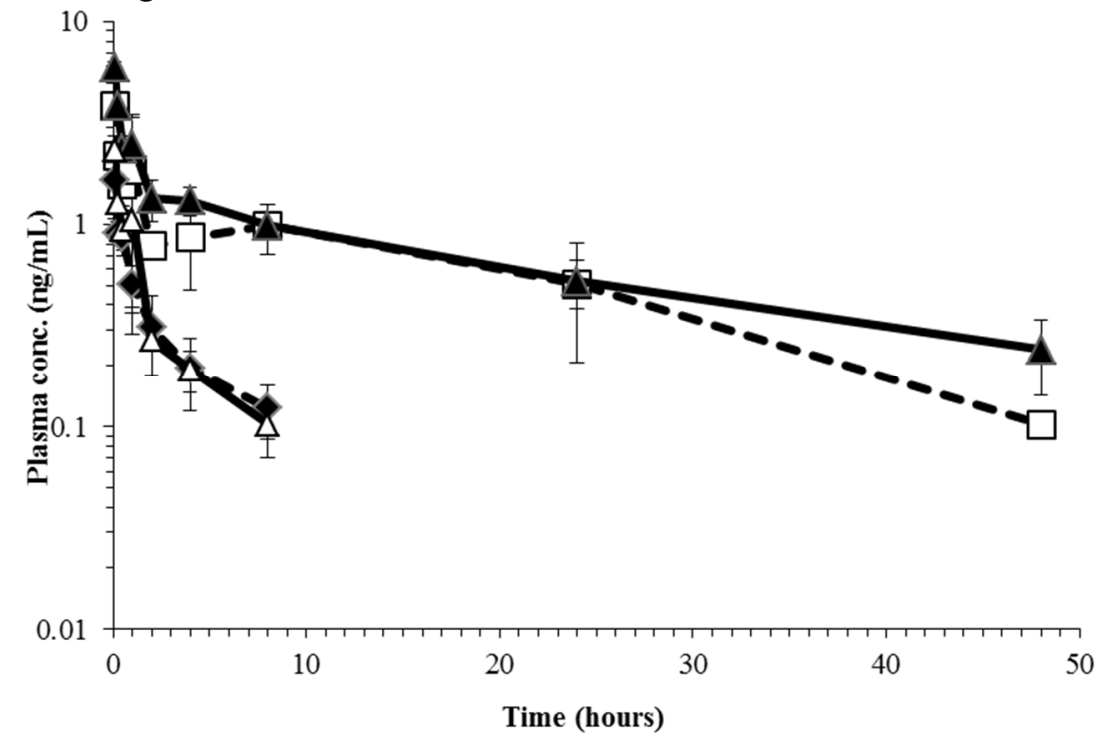

Figure 5: Pharmacokinetic profiles of plitidepsin after i.v. administration to mice of plitidepsinloaded PGA nanocapsules ( $\square$ ), PGA-PEG nanocapsules ( $\boldsymbol{\Delta})$, PEGylated nanoemulsion $(\diamond)$ and control anionic nanoemulsion $(\Delta) .($ Mean \pm S.D.; $n=3)$.

It can be noted that plitidepsin plasmatic levels achieved after the administration of drug-loaded nanocapsules are much higher than those corresponding to the control emulsions, which were below the level of detection $(0.1 \mathrm{ng} / \mathrm{mL})$ after 8 hours of administration. Similar conclusions can be drawn from Table 2, which shows the pharmacokinetic parameters associated to all formulation groups. PGA and PGA-PEG nanocapsules showed higher half-life times, lower clearance and significantly higher mean residence time (MRT) than the control emulsions (nanoemulsion or PEGylated nanoemulsion). Besides this effect in the disposition parameters, it could also be noted that the volume of distribution of the nanocapsules was larger than that of the nanoemulsions. This increase in the volume of distribution has been associated to a prolonged drug plasmatic residence time and, thus, to a facilitated access to peripheral tissues and notably to those that are hypervascularized. This is critical in order to enhance the concentration of the drug in the tumoral areas [36].

Table 2: Pharmacokinetic parameters of plitidepsin-loaded PGA, PGA-PEG nanocapsules, nanoemulsion and PEGylated nanoemulsion after a single i.v. administration to mice.

\begin{tabular}{|c|c|c|c|c|c|}
\hline Prototype & $\begin{array}{c}\text { t1/ } 2_{\beta} \\
\text { (h) }\end{array}$ & $\begin{array}{l}\operatorname{AUC}_{0 \rightarrow t} / \text { Dose } \\
(\mathrm{ng} \cdot \mathrm{h} / \mathrm{mL} / \mathrm{mg})\end{array}$ & $\begin{array}{c}\text { CLp } \\
(\mathrm{mL} / \mathrm{min} / \mathrm{kg})\end{array}$ & $\begin{array}{c}V d_{\beta} \\
(\mathrm{L} / \mathbf{k g})\end{array}$ & $\begin{array}{c}\text { MRT } \\
\text { (h) }\end{array}$ \\
\hline NCs PGA & 13.3 & 69.32 & 224.8 & 258.7 & 17.0 \\
\hline NCs PGA-PEG & 18.4 & 84.1 & 166.5 & 265.8 & 24.1 \\
\hline $\mathrm{NE}$ & 4.5 & 25.0 & 507.5 & 197.9 & 5.2 \\
\hline PEG NE & 4.2 & 29.9 & 462.8 & 168.5 & 3.9 \\
\hline
\end{tabular}

Overall, these parameters point out to a clear improvement in the pharmacokinetic behaviour of plitidepsin when encapsulated into PGA nanocapsules. This improvement in pharmacokinetics has already been observed for a variety of drug nanocarriers [7, 37-39] and has been related to the stealth properties of nanocarriers [35]. 


\section{Toxicity study}

As pointed out in the introduction, improving the efficacy/toxicity ratio of current therapies is the ultimate goal of anticancer nanomedicines. In this study, the toxicity of plitidepsin-loaded PGA and PGA-PEG nanocapsules was evaluated upon i.v. administration to healthy mice. Plitidepsin-loaded nanoemulsion and plitidepsin-loaded PEGylated nanoemulsion were also studied to compare with PGA and PGA-PEG coated systems. Plitidepsin in the reference formulation (Cremophor ${ }^{\circledR} \mathrm{EL} /$ ethanol/ water $15 / 15 / 70 \mathrm{w} / \mathrm{w} / \mathrm{w}$ ) was studied as a benchmark. The toxicity for all groups was quantified by comparing the maximum tolerated dose (MTD), defined as the maximum plitidepsin dose resulting in less than $15 \%$ loss in body weight and that does not cause lethality.

The results indicated that the MTD for plitidepsin in the reference formulation was $0.3 \mathrm{mg} / \mathrm{kg}$. In contrast, the MTD for plitidepsin in PGA nanocapsules was above the maximum dose administered in this study $(1 \mathrm{mg} / \mathrm{kg})$, whereas for PGA-PEG nanocapsules, nanoemulsion and PEGylated nanoemulsion, the MTD was $0.9,0.9$ and $0.95 \mathrm{mg} / \mathrm{kg}$, respectively. Furthermore, no toxicity was observed upon administration of the unloaded nanocapsules. From these results it can be inferred that the maximum toxicity reduction was achieved for plitidepsin-loaded PGA nanocapsules (MTD more than 3 times higher than that of the reference formulation) and that the PEGylated formulations behaved similarly to the reference nanoemulsion. The limited efficacy of the PEGylated formulations as compared to a reference nanoemulsion could be seen as a controversy with the pharmacokinetic profiles and requires further investigation. A hypothesis for the reduced toxicity of the nanoemulsion formulation despite its rapid blood clearance could be related to the potential slow release properties of the nanoemulsion. Despite of this, overall these data evidence the reduced toxicity of the nano-oncological compositions developed as compared to the standard formulation approaches [40].

\section{Conclusions}

Herein we report for the first time a novel nanocarrier consisting of polyaminoacid nanocapsules and the pharmacokinetics/toxicity proof-of-principle for the anticancer drug plitidepsin. Besides their optimum pharmaceutical properties (easy production and stability), these nanocapsules exhibited highly improved biodistribution and toxicity profiles compared to the plitidepsin conventional formulation. Overall, this study highlights the promising potential of PGA nanocapsules as delivery carriers systems for anticancer drugs.

\section{Acknowledgements}

Financial support from CENIT-NANOFAR XS53 project, PharmaMar, Spain, the Ministry of Sciences and Innovation (CTQ2009-10963 and CTQ2009-14146-C02-02) and the Xunta de Galicia (Competitive Reference Groups-FEDER funds Ref. 2010/18 and 10CSA209021PR, CN2011/037) and European Commission FP7 EraNet - EuroNanoMed Program-Instituto Carlos III (Lymphotarg proyect, Ref. PS09/02670). Giovanna Lollo has a fellowship from the Ministry of Education of Spain. Marcos Garcia Fuentes acknowledges an Isidro Parga Pondal Fellowship from Xunta de Galicia.

\section{References}

1. J.H. Park, S. Lee, J.-H. Kim, K. Park, K. Kim, I.C. Kwon, Polymeric nanomedicine for cancer therapy, Progress in Polymer Science, 33 (2008) 113-137.

2. H. Gelderblom, J. Verweij, K. Nooter, A. Sparreboom, Cremophor EL: the drawbacks and advantages of vehicle selection for drug formulation, European Journal of Cancer, 37 (2001) $1590-1598$.

3. E. Blanco, A. Hsiao, A.P. Mann, M.G. Landry, F. Meric-Bernstam, M. Ferrari, Nanomedicine in cancer therapy: Innovative trends and prospects, Cancer Sci., 102 (2011) 1247-1252. 
4. G. Lollo, G. Rivera-Rodriguez, D. Torres, M.J. Alonso, Oncologic nanotherapies: current applications and future perspectives, An. Real Acad. Nac. Farm., 77 (2011) 76-98.

5. S.M. Moghimi, J. Szebeni, Stealth liposomes and long circulating nanoparticles: critical issues in pharmacokinetics, opsonization and protein-binding properties, Progress in Lipid Research, 42 (2003) 463-478.

6. Y. Malam, M. Loizidou, A.M. Seifalian, Liposomes and nanoparticles: nanosized vehicles for drug delivery in cancer, Trends in Pharmacological Sciences, 30 (2009) 592-599.

7. J. Hrkach, D. Von Hoff, M.M. Ali, E. Andrianova, J. Auer, T. Campbell, D. De Witt, M. Figa, M. Figueiredo, A. Horhota, S. Low, K. McDonnell, E. Peeke, B. Retnarajan, A. Sabnis, E. Schnipper, J.J. Song, Y.H. Song, J. Summa, D. Tompsett, G. Troiano, T. Van Geen Hoven, J. Wright, P. LoRusso, P.W. Kantoff, N.H. Bander, C. Sweeney, O.C. Farokhzad, R. Langer, S. Zale, Preclinical Development and Clinical Translation of a PSMA-Targeted Docetaxel Nanoparticle with a Differentiated Pharmacological Profile, Science Translational Medicine, 4 (2012) 128-139.

8. F. Canal, J. Sanchis, M.J. Vicent, Polymer-drug conjugates as nano-sized medicines, Current Opinion in Biotechnology, 22 (2011) 894-900.

9. C. Oerlemans, W. Bult, M. Bos, G. Storm, J.F.W. Nijsen, W.E. Hennink, Polymeric Micelles in Anticancer Therapy: Targeting, Imaging and Triggered Release, Pharm. Res., 27 (2010) 2569-2589.

10. P. Hervella, G. Lollo, F. Oyarzun-Ampuero, G. Rivera-Rodriguez ,D. Torres, M.J. Alonso, Nanocapsules as Carriers for the Transport and Targeted Delivery of Bioactive Molecules, in: Trindade T, Daniel A.L., (Ed.) Nanocomposite particles for bio-aplications: Materials and bio-interfaces, Pan Stanford Publishing, Singapore, 2011, pp. 350.

11. M.V. Lozano, H. Esteban, J. Brea, M.I. Loza, D. Torres, M.J. Alonso, Intracellular delivery of docetaxel using freeze-dried polysaccharide nanocapsules, Journal of Microencapsulation, 30 (2013) 181-188.

12. N.T. Huynh, C. Passirani, P. Saulnier, J.P. Benoit, Lipid nanocapsules: A new platform for nanomedicine, International Journal of Pharmaceutics, 379 (2009) 201-209.

13. M.J. Alonso, P. Couvreur, Historical view of the design and development of nanocarriers for overcoming biological barriers, in: A.M.J.a.C. N. (Ed.) Nanostructured Biomaterials for Overcoming Biological Barriers, The Royal Society of Chemistry Cambridge UK, (2012) pp 3-36.

14. N.T. Huynh, E. Roger, N. Lautram, J.-P. Benoit, C. Passirani, The rise and rise of stealth nanocarriers for cancer therapy: passive versus active targeting, Nanomedicine, 5 (2010) $1415-1433$.

15. D.E. Owens Iii, N.A. Peppas, Opsonization, biodistribution, and pharmacokinetics of polymeric nanoparticles, International Journal of Pharmaceutics, 307 (2006) 93-102.

16. J.V. González-Aramundiz, M.V. Lozano, A. Sousa-Herves, E. Fernandez-Megia, N. Csaba, Polypeptides and polyaminoacids in drug delivery, Expert Opinion on Drug Delivery, 9 (2012) 183-201.

17. B.C. Dash, G. Réthoré, M. Monaghan, K. Fitzgerald, W. Gallagher, A. Pandit, The influence of size and charge of chitosan/polyglutamic acid hollow spheres on cellular internalization, viability and blood compatibility, Biomaterials, 31 (2010) 8188-8197.

18. J.W. Singer, Paclitaxel poliglumex (XYOTAX $\left.{ }^{\mathrm{TM}}, \mathrm{CT}-2103\right)$ : A macromolecular taxane, Journal of Controlled Release, 109 (2005) 120-126.

19. C. Li, S. Wallace, Polymer-drug conjugates: Recent development in clinical oncology, Advanced Drug Delivery Reviews, 60 (2008) 886-898.

20. W. Song, M. Li, Z. Tang, Q. Li, Y. Yang, H. Liu, T. Duan, H. Hong, X. Chen, Methoxypoly(ethylene glycol)-block-Poly(L-glutamic acid)-Loaded Cisplatin and a Combination With iRGD for the Treatment of Non-Small-Cell Lung Cancers, Macromolecular Bioscience, 12 (2012) 1514-1523. 
21. Y. Bae, K. Kataoka, Intelligent polymeric micelles from functional poly(ethylene glycol)poly(amino acid) block copolymers, Advanced Drug Delivery Reviews, 61 (2009) 768-784.

22. M. Morille, T. Montier, P. Legras, N. Carmoy, P. Brodin, B. Pitard, J.P. Benoit, C. Passirani, Long-circulating DNA lipid nanocapsules as new vector for passive tumor targeting, Biomaterials, 31 (2010) 321-329.

23. G.-S.L. Muñoz-Alonso MJ, Martínez T, Losada A, Galmarini CM, Muñoz A., The mechanism of action of plitidepsin, Curr Opin Investig Drugs, 10 (2009) 536-542.

24. P. Calvo, C. Remuñán-López, J. Vila-Jato, M. Alonso, Development of positively charged colloidal drug carriers: Chitosan-coated polyester nanocapsules and submicron-emulsions, Colloid \&amp; Polymer Science, 275 (1997) 46-53-53.

25. P. Avilés, M.J. Guillén, P.Calvo, M.J. Alonso, D. Torres, M. García Fuentes, T. Gonzalo, Lollo G., Nanocapsules for use in pharmaceutical compositions (EP11382003.9)

26. E.F.A. Brandon, R.D. van Ooijen, R.W. Sparidans, L.L. Lázaro, A.J.R. Heck, J.H. Beijnen, J.H.M. Schellens, Structure elucidation of aplidine metabolites formed in vitro by human liver microsomes using triple quadrupole mass spectrometry, Journal of Mass Spectrometry, 40 (2005) 821-831.

27. B. Romberg, J.M. Metselaar, L. Baranyi, C.J. Snel, R. Bünger, W.E. Hennink, J. Szebeni, G. Storm, Poly(amino acid)s: Promising enzymatically degradable stealth coatings for liposomes, International Journal of Pharmaceutics, 331 (2007) 186-189.

28. K. Knop, R. Hoogenboom, D. Fischer, U.S. Schubert, Poly(ethylene glycol) in Drug Delivery: Pros and Cons as Well as Potential Alternatives, Angewandte Chemie International Edition, 49 (2010) 6288-6308.

29. M.V. Lozano, D. Torrecilla, D. Torres, A. Vidal, F. Dominguez, M.J. Alonso, Highly efficient system to deliver taxanes into tumor cells: Docetaxel-loaded chitosan oligomer colloidal carriers, Biomacromolecules, 9 (2008) 2186-2193.

30. C. Kusonwiriyawong, K. Atuah, O.H. Alpar, H.P. Merkle, E. Walter, Cationic stearylaminecontaining biodegradable microparticles for DNA delivery, Journal of Microencapsulation, 21 (2004) 25-36.

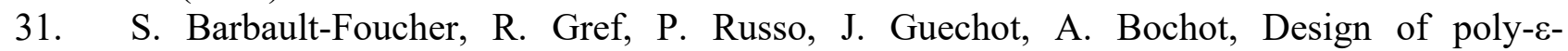
caprolactone nanospheres coated with bioadhesive hyaluronic acid for ocular delivery, $\mathrm{J}$ Control Release, 83 (2002) 365-375.

32. M. Garcia-Fuentes, D. Torres, M. Martín-Pastor, M.J. Alonso, Application of NMR Spectroscopy to the Characterization of PEG-Stabilized Lipid Nanoparticles, Langmuir, 20 (2004) 8839-8845.

33. C.E. Mora-Huertas, H. Fessi, A. Elaissari, Polymer-based nanocapsules for drug delivery, International Journal of Pharmaceutics, 385 (2010) 113-142.

34. R. Gref, A. Domb, P. Quellec, T. Blunk, R.H. Müller, J.M. Verbavatz, R. Langer, The controlled intravenous delivery of drugs using PEG-coated sterically stabilized nanospheres, Advanced Drug Delivery Reviews, 16 (1995) 215-233.

35. V. Torchilin, Tumor delivery of macromolecular drugs based on the EPR effect, Adv Drug Deliv Rev, 63 (2011) 131-135.

36. A.O. Nornoo, D.S.L. Chow, Cremophor-free intravenous microemulsions for paclitaxel: II. Stability, in vitro release and pharmacokinetics, International Journal of Pharmaceutics, 349 (2008) 117-123.

37. S.-W. Lee, M.-H. Yun, S.W. Jeong, C.-H. In, J.-Y. Kim, M.-H. Seo, C.-M. Pai, S.-O. Kim, Development of docetaxel-loaded intravenous formulation, Nanoxel-PMTM using polymerbased delivery system, J Control Release, 155 (2011) 262-271.

38. L. Wang, Z. Liu, D. Liu, C. Liu, Z. Juan, N. Zhang, Docetaxel-loaded-lipid-basednanosuspensions (DTX-LNS): Preparation, pharmacokinetics, tissue distribution and antitumor activity, International Journal of Pharmaceutics, 413 (2011) 194-201. 
39. L. Zhang, Y. He, G. Ma, C. Song, H. Sun, Paclitaxel-loaded polymeric micelles based on poly( $\varepsilon$-caprolactone)-poly(ethylene glycol)-poly( $\varepsilon$-caprolactone) triblock copolymers: in vitro and in vivo evaluation, Nanomedicine: Nanotechnology, Biology and Medicine, 8 (2012) 925-934.

40. F.K. Engels, R.A.A. Mathot, J. Verweij, Alternative drug formulations of docetaxel: a review, Anti-Cancer Drugs, 18 (2007) 95-103. 